\title{
ON BOREL DIRECTION CONCERNING SMALL FUNCTIONS
}

\author{
T. Y. PETER CHERN*
}

Abstract. In this paper, we shall prove

Theorem 1. Let $f$ be nonconstant meromorphic in $\mathbb{C}$ with finite positive order $\lambda, \lambda(r)$ be a. proximate order of $f$ and $U(r, f)=r^{\lambda(r)}$, then for each number $\alpha, 0<\alpha<\pi / 2$, there exists a. number $\varphi_{0}$ with $0 \leq \varphi_{0}<2 \pi$ such that the inequality

$$
\limsup _{r \rightarrow+\infty} \sum_{i=1}^{3} n\left(r, \varphi_{0}, \alpha, f=a_{i}(z)\right) / U(r, f)>0
$$

holds for any three distinct meromorphic functions $a_{i}(z)(i=1,2,3)$ with $T\left(r, a_{i}\right)=o(U(r, f))$, as $r \rightarrow+\infty$.

\section{Introduction and Main Results}

Let $f$ be a function meromorphic in the finite complex plane $\mathbb{C}$. We donote by $T(r, f)\left(T_{0}(r, f)\right)$ the Nevanlinna(Ahlfors-Shmizu) characteristic function of $f$. A meromorphic function $a(z)$ (including the case $f(z) \equiv c$ where $c$ in $\mathbb{C} \cup\{\infty\}$ ) is called small with respect to $f$ if $T(r, a(z))=o(T(r, f))$ as $r \rightarrow+\infty$. We let $n(r, \varphi, \alpha, f=a(z))$ be the number of roots (multiple roots being counted with their multiplicities) of the equation $f(z)=a(z)$ for $z$ in the angular domain $\Omega(r, \varphi, \alpha)=\{z:|\arg z-\varphi|<\alpha,|z|<r\}$ where $0 \leq \varphi<2 \pi, \alpha>0$.

This paper deals with the existence of the Borel directions concerning small functions for mermorphic functions of finite positive order. Using Tsuji's method, we shall mainly prove Theorem 1 stated in the abstract. Theorem 1 extends a result of Chuang [2, p.127, Corollary 5.3], there $a(z)$ are restricted over all extended complex numbers. Chuang's method is different from ours and is based on the existence of a sequence of filling disk with their roots in the works of Milloux [3] and Valiron [7].

Theorem 2. (The Existence Theorem on Borel Direction concerning small functions) If $f$ is meromorphic in $\mathbb{C}$ with order $\lambda, 0<\lambda<+\infty$, then there exists a number $\varphi_{0}$ with

Received November 15, 1995, revised September 25, 1996.

1991 Mathematics Subject Classification. Primary 30D30, 30D35

Key words and phrases. Borel direction, small function, finiter positive order.

"This research was supported in part by the NSC R. O. C. Contract 84-2121-M214-003. 
$0 \leq \varphi_{0}<2 \pi$, such that for each $\alpha>0$, the equation

$$
\limsup _{r \rightarrow+\infty} \log \left\{\sum_{i=1}^{3} n\left(r, \varphi_{0}, \alpha, f=a_{i}(z)\right)\right\} / \log r=\lambda,
$$

holds for any three distinct meromorphic functions $a_{i}(z)(i=1,2,3)$ with $^{-} T\left(r, a_{i}\right)=$ $o(T(r, f))$, a.s $r \rightarrow+\infty$.

Theorem 2 extends a result of Biernacki [1, Theorem 6.5].

Applying Theorem 1 and adapting a line of reasoning used by C. T. Chuang [2, p.128, Corollary 5.4], it is easy to obtain the following

Theorem 3. [1] If $f$ is meromorphic in $\mathbb{C}$ with finite positive order, $U(r, f)$ is given as in Theorem 1 , then there is a number $\varphi_{0}$ with $0 \leq \varphi_{0}<2 \pi$ such that for each positive number $\alpha$, the inequality

$$
\limsup _{r \rightarrow+\infty} \sum_{i=1}^{3} n\left(r, \varphi_{0}, \alpha, f=a_{i}(z)\right) / U(r, f)>0,
$$

holds for any three distinct meromorphic functions $a_{i}(z)(i=1,2,3)$ with $T\left(r, a_{i}\right)=$ $o(U(r, f))$, as $r \rightarrow+\infty$.

Theorem 3 extends a result of Valiron [8, p.34, Theorem 29.]. Since $\limsup _{r \rightarrow+\infty} \frac{T(r, f)}{U(r, f)}$ $=1$, Theorem 2 follows from Theorem 3 .

\section{The Proof of Theorem 1}

To prove Theorem 1, we need some terminologies. Let $S(r, \varphi, \alpha, f)$ be the spherical area of the image under $f$ of $\Omega(r, \varphi, \alpha)$ where $0 \leq \varphi \leq 2 \pi$. $T_{0}(r, \varphi, \alpha, f)$ be the AhlforsShimizu characteristic of $f$ associated with $S(r, \varphi, \alpha, f)$ and $N(r, \varphi, \alpha, f=a(z))$ be the integral counting function of $f$ associated with $n(r, \varphi, \alpha, f=a(z))$. Suppose that the conclusion of 'Theorem 1 is incorrect, then there exists a positive number $\alpha, 0<\alpha<\pi / 2$; for each $\varphi, o \leq \varphi<2 \pi$, there exist thrce distinct, meoromorphic functions $a_{\varphi_{j}}(j=1,2,3)$ with $T\left(r, a_{\varphi_{j}}\right)=o(U(r, f))$ such that the expression

$$
\limsup _{r \rightarrow+\infty} \sum_{j=1}^{3} n\left(r, \varphi_{0}, \alpha, \dot{f}=a_{\varphi_{j}}(z)\right) / U(r, f)=0,
$$

holds.

Since $\chi=\{(\varphi-\alpha / 4, \varphi+\alpha / 4): \varphi \in[0,2 \pi]\}$ is an open covering of the closed interval $[0,2 \pi]$ and $[0,2 \pi]$ is compact; so there exists a finite subcovering $\chi_{0}^{\prime}=\left\{\left(\varphi_{k}-\alpha / 4, \varphi_{k}+\right.\right.$ $\alpha / 4) \mid k=1, \ldots, n\}$ which covers $[0,2 \pi]$. 
For each positive integer $k, 1 \leq k \leq n$, we put

$$
F_{k}(z)=\left(f(z)-a_{\varphi_{k 1}}(z)\right)\left(a_{\varphi_{k 3}}(z)-a_{\varphi_{k 2}}(z)\right) /\left(f(z)-a_{\varphi_{k 2}}(z)\right)\left(a_{\varphi_{k 3}}(z)-a_{\varphi_{k 1}}(z)\right),
$$

where $a_{\varphi_{k j}}(z),(j=1,2,3)$ depending on $\varphi_{k}$ and $\alpha$ and satisfying the expression (2.1). The function $f$ can be written as

$$
f=\left(g_{\varphi_{k 1}} F_{k}+g_{\varphi_{k 2}} /\left(g_{\varphi_{k 3}} F_{k}+g_{\varphi_{k 4}}\right) .\right.
$$

For above expression (2.3), applying [6, Lemma, p.277], we have

$$
S\left(r, \varphi_{k}, \alpha / 4, f\right) \leq 27 S\left(64 r, \varphi_{k}, \alpha / 2, F_{k}\right)+o\left(\int_{1}^{128 r}\left(\sum_{j=1}^{4} T\left(t, \dot{g}_{\varphi_{k j}}\right) / t\right) d t\right) .
$$

Dividing two sides of the above inequality (2.4) by $r$, and then integrating them to $r$, and then applying [6, Theorem VII.8, p.272], we have

$$
\begin{aligned}
T_{0}\left(r, \varphi_{k}, \alpha / 4, f\right) & \leq 27 T_{0}\left(64 r, \varphi_{k}, \alpha / 2, F_{k}\right)+o(U(r, f)) \\
& \leq 81 \sum_{j=1}^{3} N\left(128 r, \varphi_{k}, \alpha, F_{k}=b_{j}\right)+o(U(r, f))
\end{aligned}
$$

where $b_{i}=0, b_{2}=1, b_{3}=\infty$.

Since $\chi_{0}$ covers $[0,2 \pi]$, we have

$$
\begin{aligned}
T_{0}(r, f) & \leq \sum_{k=1}^{n} T_{0}\left(r, \varphi_{k}, \alpha / 4, f\right) \\
& \leq 81 \sum_{k=1}^{n} \sum_{j=1}^{3} N\left(128 r, \varphi_{k}, \alpha, F_{k}=b_{j}\right)+o(U(r, f)) \\
& =81 \sum_{k=1}^{n} \sum_{j=1}^{3} N\left(128 r, \varphi_{k}, \alpha, f=a_{\varphi_{k j}}\right)+o(U(r, f)) .
\end{aligned}
$$

Dividing two sides of the above inequality $(2.6)$ by $U(r, f)$, then taking limsup $r \rightarrow+$, and then applying the L' Hopital Rule we have

$$
\begin{aligned}
\limsup _{r \rightarrow+\infty} T_{0}(r, f) & / U(r, f) \leq 81 \sum_{k=1}^{n} \limsup _{r \rightarrow+\infty} \sum_{j=1}^{3} N\left(128 r, \varphi_{k}, \alpha, f=a_{\varphi_{k j}}\right) / U(r, f) \\
& =81 \sum_{k=1}^{n}(128)^{\lambda} \limsup _{r \rightarrow+\infty} \sum_{j=1}^{3} N\left(r, \varphi_{k}, \alpha, f=a_{\varphi_{k j}}\right) / U(r, f) \\
& \leq 81(128)^{\lambda} \sum_{k=1}^{n} \limsup _{r \rightarrow+\infty} \sum_{j=1}^{3}(1 / \lambda) n\left(r, \varphi_{k}, \alpha, f=a_{\varphi_{k j}}\right) / U(r, f)=0 .
\end{aligned}
$$


Above result contradicts $\lim \sup _{r \rightarrow+\infty} T(r, f) / U(r, f)=1$, and $T_{0}(r, f) \sim T(r, f)$. This completes the proof of Theorem 1 .

\section{References}

[1] M. Biernacki, "Sur les directions de Borel des functions meromorphes," Acta Math., 56 (1930), 197-204.

[2] C. 'T. Chuang, Sigular Direction of Meromorphic Functions (in Chinese), Science Iress, Beijing, 1982.

[3] H. Milloux, "Le theoreme de Picard, suites de functions holomorphes; functions meromorphes et functions entieres," J. dc Math., 3 (1924), 345-401.

[4] X. Pang, "On the singular dircction of mcromorphic function" (in Chinesc), Advances in Mathematics (China), 16 (3) (1987), 309-315.

[5] M. Tsuji, "On Borel's directions of meromorphic functions of finite order, III, Kodai Math. Sem. Rep., (1950), 104-108.

[6] M. Tsuji, Potential Theory in Modern Function Theory, Chelsca Publ., N. Y., 1975.

[7] G. Valiron, "Recherches sur le theoreme de M. Borel dans la theorie des functions meromorphes," Acla Math. 52 (1928), 67-92.

[8] G. Valiron, Directions de Borcl des functions meromorphes, Memor. Sci. Math., Fasc. 89, Press, 1938.

Department of Applied Mathemalics, aohsiung Polytechnic Institute, Ta-Isu Hsiang, Kaohsiung County, Taiwan 84008 R.O.C. 